\title{
Design and Development of Social Distancing Smart Wear
}

\author{
V Parthasarathi, M Parthiban \\ \{*mpn.fashion@psgtech.ac.in,vps.fashion@psgtech.ac.in \} \\ Assistant Professor (Sel.Gr),Department of Fashion Technology, PSG College of \\ Technology,Tamilnadu ,Coimbatore-641004,India ${ }^{1,2}$
}

\begin{abstract}
Covid-19 is a dreadful virus. Since December 2019, the cases around the world are over 15 million with the death rate of $1.2 \%$ till April 2021 that staggered the nation worldwide. Due to its contagious nature, it is considered as a pandemic disease by World Health Organization (WHO). Despite all protection, the main advice is to follow social distancing among people. The higher level of exposure on social distancing will decline the curve by $70 \%$ to $90 \%$. This research work is to develop a social distancing device as a wearable technology, which will be a great contribution from fashion and textile side to curb the disease firmly. It is achieved by embedding electronics with fashion and designed in such a way to make it as a wearable technology- to give an alarm sound or a blink on arm side and waist bag made as a solution to this problem. To bring it to successful development, three ways were approached for choosing the best one for the product. The traditional arduino nano with sensor, the radio communication using micro bit, and the simulation from image processing methods have been used and tested properly in different conditions. All methods have been programmed and developed not only as per government prescribed norms but also from consumer's point of view. The arm side bag product was fabricated and constructed in a proper way to give alert along with probability. The prototypes and fabrics were validated and experimented thoroughly according to the requirements. Thus, this concept and idea will have a capability to keep people to maintain safe distance of 6 feet to 8 feet among people. By wearing this electronic wear which will create an impact of alert system on social distancing for changing the frightened situation go normal, and can turn the world free from the deadly virus.
\end{abstract}

Keywords: Covid-19, sensors, smart clothing, social distancing, radio communication, wearable electronics

\section{Introduction}

In worldwide recorded over 15 million confirmed cases due to COVID-19, from December of 2019 till April 2021. Worldwide organization had declared the occurrence of pandemic in March 2020. As results, old-age individuals, underneath health condition individuals these were the individuals were notably stricken by COVID nineteen this information is given by china. Just about eighty one of deaths were occurred for adulthood sixty years, only $0.1 \%$ deaths were occurred middle aged individuals nineteen years (CDC response team, 2020) due to COVID nineteen principally affected sectors is cordial reception and business sector [1].

These each sectors were affected in worldwide as a result of they were the individuals most of the time contact with people in order that they have to be compelled to take some strict measures. In order 
that they have to be compelled to take safety measures and if they did not take safety measures they would be stricken by COVID. (International journal of cordial reception, 2020) In urban center, Hubei Province, China, in December 2019, associate in progress occurrence of respiratory illness related to a unique corona virus, severe acute metabolic process syndrome corona virus two, was according. Infections unfold across China and different countries round the world, within the following weeks. Timely recognition was allowed to require action by the new virus and shared the microorganism citron sequence to the globe. The World Health Organization declared the outbreak a Public Health Emergency of International Concern on in 30, 2020.

The WHO named the disease caused by the novel corona virus "corona virus disease 2019" on Feb 12, 2020. Chinese counterparts to try to contain the outbreak. A group of international experts, with a range of specializations have worked at present, development of a real-time reverse-transcription polymerase chain reaction assay for COVID-19 used in clinics. Although the reference standard for making a definitive diagnosis of COVID-19 infection was remained by RT-PCR, Prompting the diagnosis of infected patients the high false-negative rate and the unavailability of the RT-PCR assay in the early stage of the outbreak restricted.

Important role in fighting this infectious disease have been played by audio logical examinations, especially thin-slice chest CT. Chest CT can help identify the early phase lungs infection and prompt larger public health surveillance and response systems Chest CT findings have been recommended as major evidence for confirmed clinical diagnosis in Hubei, China happened currently [3].

The addition of chest $\mathrm{CT}$ for designation resulted in fourteen 840 confirmed new cases rumored on February thirteen, 2020. Comprehensive and timely review of the role of radiology in fighting COVID19 remains imperative and necessary. Individual's trade off the utility advantage of group action against the inner and external health risks that go with social interactions throughout a plague explores by developing a quantitative framework. External targets and so compare its predictions with daily knowledge on group action, fatalities, and also the calculable effective copy range $\mathrm{R}$ ( $\mathrm{t}$ ) from the COVID-19 pandemic in March-June 2020, calibrated by the model.

We have a tendency to discover in USA knowledge, whereas the laissez- faire equilibrium is in step with abundant of the decline in group action, optimum policy additional imposes immediate and extremely persistent social distancing. Neither equilibrium nor optimum social distancing is very restrictive, within the sense that the effective copy range ne'er falls so much below one, notably. The expected price of COVID-19 within the USA is substantial, $\$ 12,700$ within the capitalistic equilibrium and $\$ 8,100$ per person below Associate in nursing optimum policy. This huge welfare gain by shifting the composition of prices from fatalities to persistent social distancing that is generated by optimum policy [4].

Safety measures of COVID are wearing mask, social distancing, and sanitizing, washing hands often. Here we have a tendency to mention concerning social distancing. Social distancing is that the vital answer for COVID nineteen. Whereas we have a tendency to go out with a gap between 2 folks is a pair of meters. we've got to cut back time for going outdoors as a result of once folks getting ready to people it will have an effect on North American country as a result of spreading of COVID [5].

In end of 2020, COVID-19 vaccines were successfully developed and tested in time frames previously thought impossible. The first findings came from two mRNA COVID-19 vaccines, both of which had high efficacy (94-95\%). 1\&2; the low temperature storage requirements of these two vaccines is a drawback. While there have been other COVID-19 vaccines produced to date that use viral vectors, subunit particles, or inactive virus, this is the first of its kind. After a time of relative viral genetic stability, the recent emergence of SARS-CoV-2 variants is cause for concern, as several new escape variants could emerge in the future, leading to a serious epidemic rebound, as seen in many global countries. Increased viral transmission provides further chances for SARS-CoV-2 variants to evolve. 
As a result, the pandemic would only be ended when vaccines would be successful [6]. Finally, vaccines have had been readily distributed, affordable, and conveniently usable on a global scale. Although WHO has called for greater equity and stronger support for the COVAX initiative and its mandate of equal vaccine access, particularly for resource-limited countries, many high-income countries have purchased vaccine doses, often close to nine doses per person. The vaccination program should be advertised in such a way to create more awareness on controlling and ending the dreadful pandemic [7].

Despite the arrival of vaccines, lockdown has been steadily removed in many countries; once again, the effect of the virus is posing threats to many people, especially in developing nations, and the virus has grown into a community of distinct strains that cause much more serious health issues than its predecessor. As a result, the government has debated current scenario options and has begun to include informing people about vaccines and following other preventive measures [8].

Social distancing measure is one of first tool to scale back the transmission of the severe acute metabolic process syndrome corona virus two (SARS-CoV-2) viruses that causes corona virus no wellness 2019, within the absence of an immunizing agent. Financial gain varies that we tend to show that social distancing following United States state-level emergency declarations considerably. Mobile device location pings, we discover that wealthier areas decreased quality considerably over poorer areas, and this general pattern holds across financial gain amount, knowledge sources, and quality measures derived by quality measures [9]. style specializing in behavior succeeding state emergency orders, we tend to document a reversal within the ordering of social distancing by financial gain employed by event study: rich areas went from most mobile before the pandemic to least mobile, while, for multiple measures, the poorest areas went from least mobile to most.

Lower financial gain communities have higher levels of pre-existing health conditions and lower access to tending showed by previous analysis. Combining this with our core finding-that lower financial gain communities exhibit less social distancing - suggests a double burden of the COVID-19 pandemic with stark spacing implications [10].

On the account of the tragic state of scenario, our core project plan is to design and develop a social distancing sensible wear with options of comfort property for all individuals as well as to different stream professionals. This idea can produce an alert system for people who wear this technology to maintain their safe distance of 1.5 meters to 2 meters as per government guidelines. The main objective of our project is to choose the best material, the comfort style and therefore the applicable ways to engineer the model in thriving manner to provide a good answer to this state of current problem in a form of electronic and fashion product with portability [11].

Corona is a deadly virus, which started its spread during the end of 2019, in China. Wuhan of China is the first victim to the demon. Later, it started having its effect world-wide, and the WHO declared it to be a global pandemic, COVID -19. It had its effect on human of all age categories, irrespective of their age, race $\&$ health stature. It is said that the virus mainly affects one's respiratory system causing fever, cold, cough \& pneumonia. Also, the virus is spread through contact. Hence, governments of all the nations throughout the world were forced to announce closure of global borders, lockdown \& socialdistancing. A minimum of $6 \mathrm{ft}-8 \mathrm{ft}$ distance has to be kept from person to person, in order to curb the spread of the virus. People were instructed to wear face mask, sanitize their hands, and quarantine themselves in case of any symptoms [12].

\section{Impact Of COVID-19}

Covid-19 created great psychologically affect on people and also it affects the people and also it affects the people economy. To reduce the covid cases there are more ways that can be followed by the 
people it helps them to be get affected from the virus. The ways can be followed by the people are social distancing, self-isolation at home, closure of institutions \& public facilities, restrictions on mobility, even lock-downs. The corona virus SARS-COV-2 is the threat of all human lives it spreads respiratory illness and it leads to life deaths. The early stages of the covid the medical interventions, vaccines are all extremely limited (or) non- existent. Hence local, national, global government and public office had taken the difficult decision to implement the non-medical interventions. The non- medical interventions helps people from spreading of covid. [13].

\section{Social Distancing}

Social Distancing is commonly known as public distancing. Commonly this distancing is to maintain a safe space between each other by restricting people from physical contact, in order to reduce the containment of the virus, as the same was easily spreadable. Social distancing reduces the physical interactions and it is the first step defense fight against the pandemic. [14].

COVID-19 highly spreads during the physical contact with the people; it mostly affects the people with low immunity. Here the physical contact is that hand shaking, cash handling, purchasing etc. Alternatives can be done for hand shaking, hugging and the gestures like Namaste, thumbs up, hands on heart, wave, bow, salute, elbow bump etc.., to maintain social distancing and without physical contact $[15]$.

Social distancing is more important for all the people. If an affected person comes in the public and he sneezes or coughs the droplets may have millions of virus that will affect the people up to maximum 3 feet distance get affected [16].

According to the report distributed by world wellbeing association, the infection may spread because of close contact. This COVID 19 may spread through contacting the contaminated surface. The essential focal point of our work is to caution individuals when they intentionally or unwittingly pass the boundary of a S2D limit that is 6 feet or 2 meters. The proposed model is the mix of closeness sensor and a disturbing model. To plan this social separating gadget the idea of closeness sensor is utilized [17].

In view of attractive field wearable vicinity detecting framework can be assessed. The fundamental actual standard and proposed convention for the distinguishing proof and coordination of the transmitter can be examined. The proposed framework is substantially more dependable than the broadly utilized Bluetooth based methodology especially with regards to recognize distance above and beneath the two meter limit because of attractive field actual property [18].

Recurrence of contact and total time that have spent together is named by each edge. For example, expect a positive case is figured out at time $\mathrm{T}$. The hub with way distance one from the positive hub are changed over to red. The redness of changed over light is straightforwardly corresponding to the recurrence and term of their contact to the positive hub. The hubs which are connected to positive hub through contact are additionally plausible case with lower likelihood and will be stamped yellow. The power of the yellow tone is contrarily relative to the way distinction from red hubs [19].

While travelling one can detect red and yellow gadget to keep up the social distancing with notice signals. Rehearsing safe separation from others if close by red/yellow devise exist to straighten the contamination and it is significant for rehearsing. [20]. It is a transmission and discovery of radio signals comprising of electromagnetic waves movement through the air in an orderly fashion or by reflection from the ionosphere or from a satellite [21]. Electromagnetic radiations share two principle properties practically speaking they are light and radio waves. Both can go through space in straight line at speed of 300,000,000 meters/second and amplitudes differ consistently with time [22]. 
The wave is comprised of electric and attractive fields; it vibrates commonly at right points to one another in space. While these two fields work simultaneously on schedule, they are known to be in time stage. Both come to their maxima\& minima together and if both go through zero together [23]. Wellspring of energy expands the region over which the electric and attractive energy spread increments; however the accessible energy per unit territory is decreased [24]. The main objectives of this research works are design and develop a social distancing device as a wearable. To take a survey to collect consumer suggestions on developing the project. To pick durable electronic components and fabrics with best textures and comfort properties. To ensure it meets all the requirements as per government norms as to maintain safe distance 1 to 2 meters. To blend the device onto the wear. to approve the item altogether by thorough testing and validation process.

\section{Materials And Methods}

We have conducted a pre-project survey so as to help in deciding the materials and fabrics for the development of the social distancing smart wear and the final outcome of the project is based on the consumer responses who had taken the survey. The survey had total number of four questions with multiple choices on different clothing and comfort concerning questions. Figure 1 shows the research work methodology.

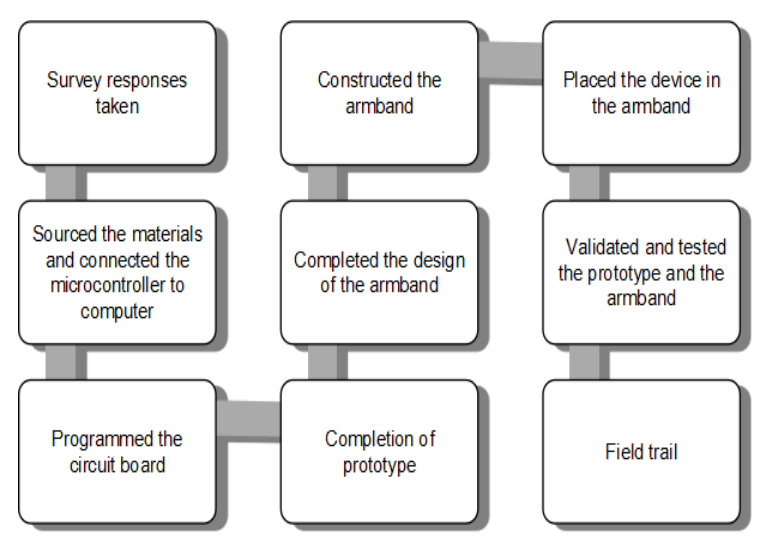

Fig 1 Research work flow process

An arduino-nano, A ultrasonic sensor, A buzzer and A LED, Connecting wires, battery components that we used to make the prototype of arduino-nano with sensor model. Each components image is shown in figure 2 .

\section{SPECIFICATIONS}

- $\quad$ Model: Arduino Nano V 3.0

- Type: Microcontroller board

- Material: Carbon fiber

- Width: $4.5 \mathrm{~cm}$

- Height: $1.8 \mathrm{~cm}$

- Weight: $7 \mathrm{~g}$

- Power: DC 
- $\quad$ ATmega328 Operating Voltage (logic level):5V Input Voltage (recommended):

- $\quad 7 \mathrm{~V} \sim 12 \mathrm{~V}$ Input Voltage (limits):

- $\quad 6 \mathrm{~V} \sim 20 \mathrm{~V}$ Digital I/O Pins: 14 (of which 6 provide PWM output)

- Analog Input Pins: 8 DC

- Current per I/O Pin: 40mA

- $\quad$ Flash Memory: 32KB (ATmega328) (of which $2 \mathrm{~KB}$ used by boot loader)

- $\quad$ SRAM: 2KB (ATmega328)

- $\quad$ EEPROM: 1KB (ATmega328)

- $\quad$ Clock Speed: $16 \mathrm{MHz}$

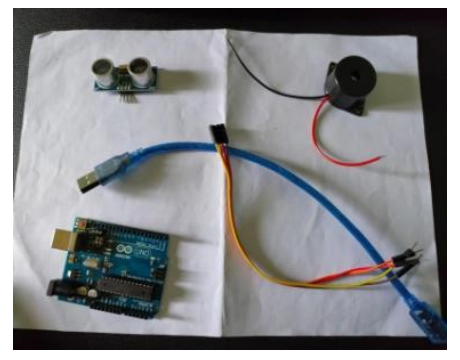

Fig 2 Components used in circuit

The entire circuit is connected to the computer through USB cable and programmed it which the image is shown in Fig 3.

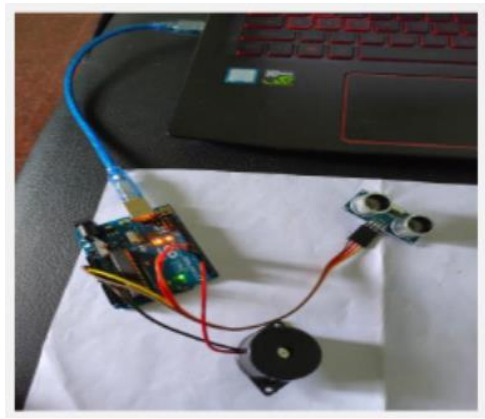

Fig 3 Circuit setup

Radio communication is an evergreen technology which we have implemented using the right components, and programmed according to the essential requirements. The working process is based on transmitting radio signals between transmitter and receiver. The flow chart is shown Fig 4. 


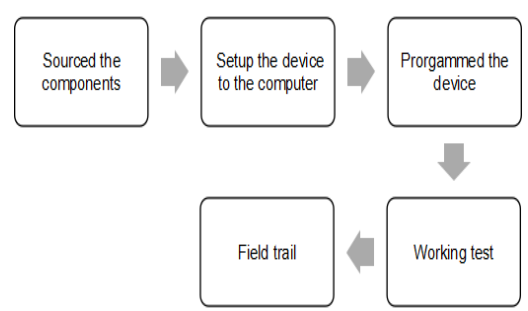

Fig 4 Radio communication flow

We sourced two micro: bits for radio communication method which the micro: bit is a compact sized programmable circuit board designed and developed by BBC which is embedded with plenty of sensors and other ports to create and develop enormous number of project prototypes that can bring many innovations to the world.

\section{Specifications of the Micro:Bit (BBC2546862)}

- Brand : Micro: bit

- Model name:: Bit go

- Company : BBC (British Broadcasting Corporation)

- Colour: Original black

- $\quad$ Build Factor: Stick type

- Number of quantity: 2

- $\quad$ Product weight: $9 \mathrm{~g}$

- Dimensions : $4 \mathrm{~cm} * 5 \mathrm{~cm} * 0.2 \mathrm{~cm}$ (Length* Breadth * Thickness)

- Battery requirement $: 2 *$ AAA batteries (for each)

- Output Interface : Buttons and $5 * 5$ LED matrix

- Processor port : DIP

- $\quad$ Processor architecture: 32 bit

- Type of Processor: ARM (Advance RISC Machines) Cortex

- Graphics Interface: Accelerated Graphics Port (AGP)

- $\quad$ RAM and Memory flash: $16 \mathrm{~K}$ and $256 \mathrm{k}$

- Wireless Interface :Radio and Bluetooth

- Inbuilt sensors: Temperature, Accelerometer and Compass

- Connecting port: USB A to Micro USB and JST connector

- Total Voltage: $12 \mathrm{v}$

Though the size of micro: bit is compact, its features and architecture is enormous in number which is shown below in Fig 5 . 


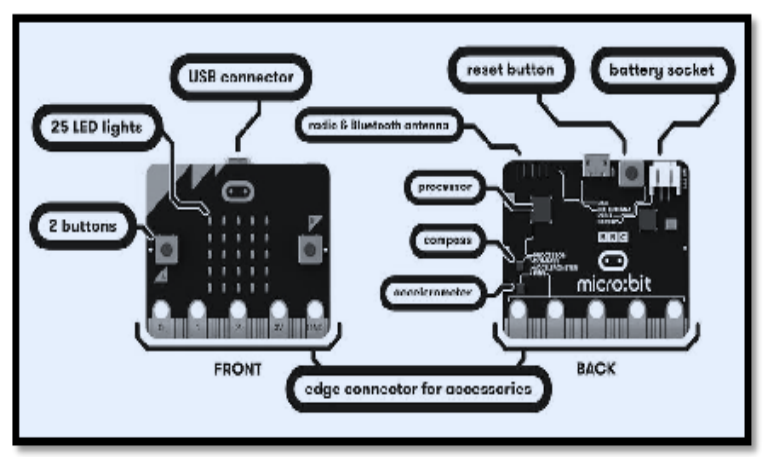

Fig. 5 Design of Micro: Bit

Each micro: bit was connected firstly through USB cable to the computer for programming the device for giving the desired output. The connection setup was not complex, as it has everything which needed for the final computerized output. It was programmed using Blocky code made by make-code website that is integrated development area for commanding the device respectively. The command was created on both devices separately in such a way to show the vital output through altering the radio signals between the two devices.

\section{Pouch Design}

For placing the gadget it is necessary to know about the product and basic requirements of the product that we had chosen. The product can be placed anywhere in our body according to the user convenience either around the waist or arm area.

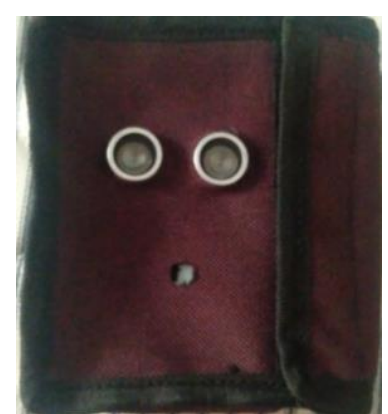

Fig 6 Device Pouch

\section{Product Validation And Testing}

For product validation the end product needs to undergo certain tests and the list of tests which should be taken are mentioned in the table given below. All images of each testing are shown table 1 .

TABLE 1

Testing Standards

\begin{tabular}{|l|l|}
\hline Name of the Test & Testing Standards \\
\hline Stiffness & ISO 6490 \\
\hline Tearing strength & ISO 13937-1 \\
\hline Abrasion resistance & ISO 12947-1 \\
\hline
\end{tabular}




\begin{tabular}{|l|l|} 
Pilling resistance & ISO 12945-1 \\
\hline
\end{tabular}

\section{Results And Discussion}

\subsection{Survey Report}

As a result of the survey response, it assisted us in deciding the design and framing the methodology to complete the prototype along with all the desirable properties that had to get added to fulfill the project outcome. In the current situation, the second wave of Covid is started in 2021, the questions helped in creating the awareness on social distancing wearable. Adhering to it, we got to know about people's interest in using a device for maintaining safe distance. The Product is designed in such a way to meet essential requirements, kind of alert system and the challenges they might face, if they wore any electronic wearable. Finally, we related the survey questions with our completed models. The results of the survey responses are shown in figure 7. Figure 7 depicts what kind of alert system people might want from the wearable and what additional things could be added based on consumers preference

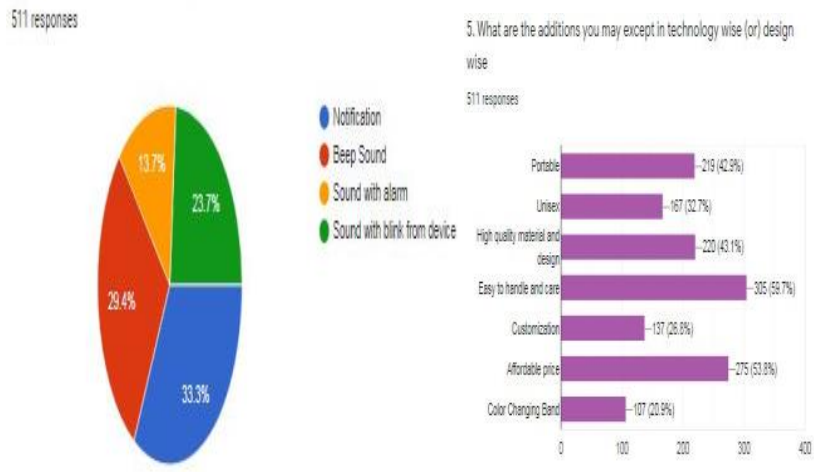

Fig 7 Survey report

TABLE II

PRODUCT VALIDATION AND TESTING RESULTS

\begin{tabular}{|c|c|c|c|}
\hline Tests & $\begin{array}{l}\text { Stan } \\
\text { dards }\end{array}$ & es $\quad$ Valu & $\begin{array}{l}\text { Acceptabil } \\
\text { ity }\end{array}$ \\
\hline $\begin{array}{l}\quad \text { Teari } \\
\text { ng } \\
\text { strength }\end{array}$ & $\begin{array}{l}\text { ISO- } \\
13937-1\end{array}$ & $\begin{array}{ll} & \text { War } \\
\text { p- } & 1.4592 \\
\text { kgf } & \\
& \text { Wef } \\
\text { t- } & 1.3348 \\
\text { kgf } & \end{array}$ & $\begin{array}{l}\quad \text { Results are } \\
\text { in } \\
\text { accepted range. } \\
\text { Since this fabric } \\
\text { was stitched } \\
\text { along with } \\
\text { outer material, } \\
\text { the tearing } \\
\text { strength } \\
\text { requirement is } \\
\text { medium }\end{array}$ \\
\hline $\mathrm{M}^{\mathrm{GS}}$ & - & $\mathrm{g} / \mathrm{m}^{\wedge} 2^{75}$ & \begin{tabular}{lr}
\multicolumn{2}{c}{ Accepted } \\
range & which \\
can & be
\end{tabular} \\
\hline
\end{tabular}




\begin{tabular}{|c|c|c|c|}
\hline & & & commercialized \\
\hline $\begin{array}{l}\text { Thic } \\
\text { kness }\end{array}$ & $\begin{array}{l}\text { AST } \\
\text { M } \\
\text { D1777- } \\
96\end{array}$ & $\mathrm{~mm}$ & \begin{tabular}{lr}
\multicolumn{2}{c}{ Accepted } \\
range which \\
can be \\
commercialized
\end{tabular} \\
\hline $\begin{array}{r}\text { Stiff } \\
\text { ness test }\end{array}$ & $6490^{\text {ISO- }}$ & $\begin{array}{l}\quad 196 . \\
98 \\
\text { mg.cm }\end{array}$ & $\begin{array}{l}\text { Accepted } \\
\text { range in } \\
\text { medium level, } \\
\text { according to the } \\
\text { bag commercial } \\
\text { requirement }\end{array}$ \\
\hline
\end{tabular}

\subsection{Coding}

The coding is done on Arduino IDE (Integrated development) software using C-language and used "if and or else" condition. All these processes are controlled by microcontroller followed by ultrasonic sensor that we programmed in an appropriate way to give the desired output. The coding is written below and the schematic diagram of arduinonano is shown in Figure 8.

constint MOTION_SENSOR_PIN = 7; // Arduino pin connected to the OUTPUT pin of motion sensor constint BUZZER_PIN = 3; // Arduino pin connected to Buzzer's pin

intmotionStateCurrent $=\mathrm{LOW}$;// current state of motion sensor's pin

intmotionStatePrevious $=$ LOW; // previous state of motion sensor's pin

void $\operatorname{setup}()\{$

Serial.begin(9600); // initialize serial

pinMode(MOTION_SENSOR_PIN, INPUT); // set arduino pin to input mode pinMode(BUZZER_PIN, OUTPUT); // set arduino pin to output mode \}

void $\operatorname{loop}()\{$

motionStatePrevious = motionStateCurrent; // store old state

motionStateCurrent $=$ digitalRead(MOTION_SENSOR_PIN); // read new state

if $($ motionStatePrevious $==$ LOW \&\&motionStateCurrent $==$ HIGH $)\{/ /$ pin state change: LOW $->$ HIGH

Serial.println("Motion detected!");

digitalWrite(BUZZER_PIN, HIGH); // turn on

\}

else

if $($ motionStatePrevious $==$ HIGH $\& \&$ motionStateCurrent $==$ LOW $)\{/ /$ pin state change: HIGH $\rightarrow$ LOW

Serial.println("Motion stopped!");

digitalWrite(BUZZER_PIN, LOW); // turn off

\} 


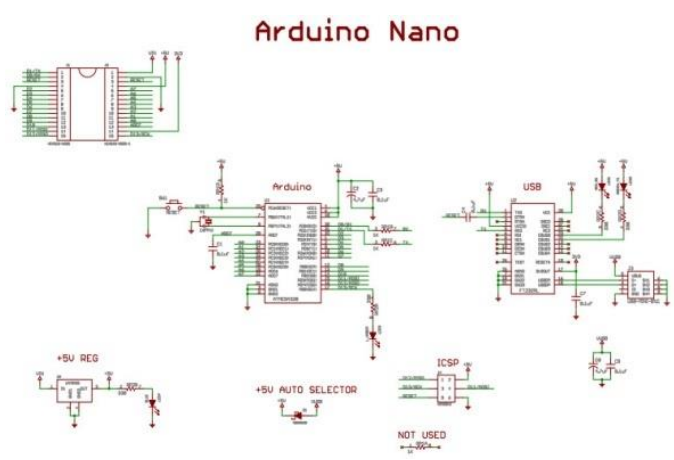

Fig 8 Schematic diagram of Arduino Nano

The end use is simple as just to wear the waist bag at the belt area. Since the material was constructed in a way to meet clothing requirements.

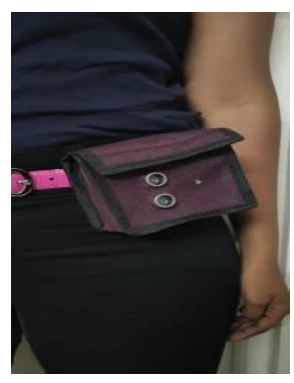

Fig 9 Prototype

The waist bag with the prototype inside is worn by the model. Micro: bit is designed in such a way to meet all consumer requirements along with the Compact size, Easy portability, comfortable and light weight.

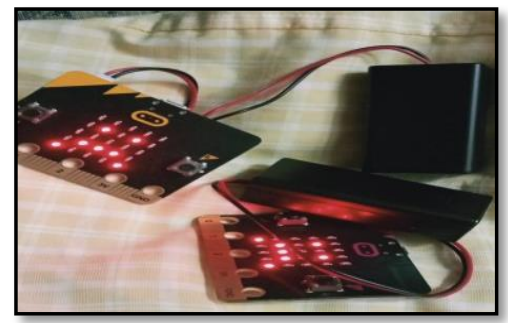

Fig 10 Final prototype of Micro: Bit

\section{CONCLUSION}

This developed prototype wear has a potential to curb the deadly pandemic situation into normal through wearable social distancing system. It will have a good market value centered for creating a safe environment. This electronic wearable must ensure people safe distance of 6 feet to 8 feet through alert system along with portability and easy to care. We recommend the radio communication method, as of ease of use and compact in size, as well as based on extensive and deliberative experimentation 
approach. Everyone must follow safety norms as per government and health guidelines by insisting people to wear the social distancing wear to be safe and protective.

\section{REFERENCES}

[1] A. Ghosh, "Nano-clay particle as Textile Coating", Int. J. Eng. Sci. Technol., vol. 11, pp.34-36, May 2011.

[2] K. M. B. Jansen, "Smart textiles: how electronics merge into our clothing", Int. Conf. Therm. Mech. Multiphys. Simul. Exp. Microelectron.Microsyst., EurosimE. 2019,

[3] H. Mohapatra, A.M.Rath, "Social Distancing Alarming Through Proximity Sensory for COVID-19", 2020.

[4] R. R. Nadikattu et al., "Novel economical social distancing smart device for Covid19", Int. J. Elect. Eng. Technol., vol. 11, no. 04, pp. 204-217, Jun. 2020.

[5] S. Bian et al., "Social Distance Monitor with a Wearable Magnetic Field Proximity Sensor", Sensors, vol. 5101, no. 20, pp.1-24, Sep. 2020

[6] A. K. Tripathy et al., "Easy-Band: A Wearable for Safety-Aware Mobility During Pandemic Outbreak”, IEEE, pp. $57-61$, Sep. 2020.

[7] A. Surve, "Social Distancing- Covid 19 solution Apron with Sticks: Simple and Low-cost", 2020.

[8] S. Sun et al., "Using Smart-phones and Wearable Devices to Monitor Behavioural Changes During COVID-19”, J. Med. Internet Res. vol. 22, no. 9, Sep. 2020.

[9] Coronaviridae Study Group of the International Committee on Taxonomy of Viruses, "The species severe acute respiratory syndrome-related corona virus: classifying $2019-\mathrm{nCoV}$ and naming it SARS-CoV-2", Nat. Microbiol., vol.5, pp.536-544, Mar. 2020.

[10] Aarogya Setu mobile app. Government of India. 2021 Available: https://www.mygov.in/aarogyasetu-app/ [accessed 2021-04-29]

[11] S. V. Arx, "Slowing the Spread of Infectious Diseases Using Crowd sourced Data", Covid Watch whitepaper, Apr. 2020. [Online]. Available: https://blog.covidwatch.org/en/covid-watchwhitepaper-using-crowdsourced-data-to-slow-virus-spread

[12] A. Parate, D. Ganesan, "Detecting Eating and Smoking Behaviours Using Smart-watches", Springer International Publishing, Cham, Switzerland, pp. 175-201, 2017.

[13] L. Cadmus-Bertram, "Using Fitness Trackers in Clinical Research: What Nurse Practitioners Need to Know", J. Nurse Pract., vol. 13, no.1, pp.34-40, Jan. 2017.

[14] Kooiman et al., "Reliability and validity of ten consumer activity trackers" BMC Sports Sci. Med. Rehabil., vol.7, no.24, Oct. 2015.

[15] M. Wilson et al., "Engaging Overweight Adolescents in a Health and Fitness Program Using Wearable Activity Trackers" J. Pediatr. Health Care, vol. 31, no.4, pp. 25-34 Jul. 2017.

[16] E.C. Nelson et.al, "Health empowerment through activity trackers: An empirical smart wristband study", Comput. Hum. Behav. vol. 62, pp.364-374, Mar.2016.

[17] J. Baek et al., "BBUNS: Bluetooth Beacon-Based Underground Navigation System to Support Mine Haulage Operations", Minerals, vol.7, no. 11:228, Nov. 2017.

[18] V. K. Singh et al., "Smart PPM: An Internet of Things Based Smart Helmet Design for Potholes and Air Pollution Monitoring.", EAI, vol. 5, no. 18: e2, Apr. 2019.

[19]Espressif Systems, "ESP32 Series [Online] Available:https://www.espressif.com/sites/default/files/documentation/esp32_datasheet_en.pdf

[20] L.O. Aghenta, M. T. Iqbal, "Low-Cost, Open Source IoT-Based SCADA System Design Using Thinger.IO and ESP32 Thing", Electronics MDPI., vol. 8, no. 8: 822, Jul. 2019.

[21] A. Haleem et.al, "Effects of covid 19 pandemic in daily life" Curr. Med. Res. Pract., vol. 10, no. 2, pp. 78-79, Apr 2020.

[22] Z. Y. Zu et al., "Coronavirus Disease 2019 (COVID-19): A Perspective from China"Radiology, vol. 296, no. 2, pp.15-25, Aug2020.

[23] J. A. Weill et al., "Social distancing responses to COVID-19 emergency declarations strongly differentiated by income", PNAS, vol. 117, no. 33, pp. 19658-19660, Aug. 2020. 
[24] X. Hu et al., "Creating a safe haven during the crisis: How organizations can achieve deep compliance with COVID-19 safety measures in the hospitality industry", Int. J. Hosp. Manage., vol. 92, Aug. 2020. 